\title{
El Papel depresor del pH durante la flotación sin colector de mineral de galena conteniendo esfalerita.
}

\author{
The Role of $\mathrm{pH}$ depressant during the collertorless flotation of galena mineral containing \\ sphalerite
}

\author{
M. Reyes Pérez ${ }^{a}, I$. A. Reyes Domínguez ${ }^{b}$, M. U. Flores Guerrero ${ }^{c}, F . R$. Barrientos Hernández ${ }^{a}$, \\ M. Pérez Labra ${ }^{a}$, J. C. Juárez Tapia ${ }^{a}$ \\ aUniversidad Autónoma del Estado de Hidalgo, Área Académica de Ciencias de la Tierra y Materiales. Abasolo 600 Col. \\ Centro, Pachuca, Hidalgo, México. \\ bUniversidad Autónoma de San Luis Potosí, Instituto de Metalurgia, Av. Sierra Leona 550, Lomas 2ª Sección 78210 San \\ Luis Potosí, S.L.P., México. \\ 'Universidad Tecnológica de Tulancingo. Área de Electromecánica Industrial. Camino a Ahuehuetitla \#301 Col. Las Presas, \\ Tulancingo, Hidalgo, México 43642.
}

\begin{abstract}
:
The recovery of the galena mineral $(\mathrm{PbS}$ ) during the collectorless flotation, decreases with the increase $\mathrm{pH}$ and with the decrease in the pulp potential $E h(\mathrm{mV})$ referred to the standard hydrogen electrode. The depression of PbS, during flotation, is attributed to the superficial state that it acquires in alkaline environments, characterized by FTIR, which consists of intense binding bands of covalent species such as; the free sulphate ion with a wavelength of $1097 \mathrm{~cm}^{-1}$, sulfo oxides $\mathrm{S}=\mathrm{O}$ at $1400 \mathrm{~cm}^{-1}$, indicating the oxidation of the sulphur of the last atomic layer and hydroxyl ion $\mathrm{OH}^{-}$at $1638 \mathrm{~cm}^{-1}$, are formed, in addition, weak absorption bands of the $\mathrm{Pb}-\mathrm{O}$ bonds. While when galena recovery is optimal $75 \% \mathrm{w} / \mathrm{w}$, the surface sulphur combines with the metal forming monodentate coordinated sulphates with three absorption bands, as well as $\mathrm{Pb}-\mathrm{OH}$ bonds of lead hydroxide $\mathrm{Pb}(\mathrm{OH})_{2}$ and $\mathrm{Pb}-\mathrm{O}$ bonds, of $\mathrm{PbO}_{2}$.
\end{abstract}

Keywords:

Galena, collectorless flotation, depressor, oxidation, $\mathrm{pH}$

\begin{abstract}
Resumen:
La recuperación del mineral de galena (PbS) durante la flotación sin colector, desciende con el incremento del pH y con la disminución del potencial de pulpa Eh (mV) referido al electrodo estándar de hidrogeno. La depresión del PbS, durante la flotación, se atribuye al estado superficial que adquiere en ambientes alcalinos, caracterizada por FTIR, el cual consiste de intensas bandas de enlace de especies covalentes tales como; el ion sulfato libre con una longitud de onda en $1097 \mathrm{~cm}^{-1}$, sulfo óxidos $\mathrm{S}=\mathrm{O}$ en $1400 \mathrm{~cm}^{-1}$, indicando la oxidación del azufre de la última capa atómica y ion hidroxilo $\mathrm{OH}^{-}$en $1638 \mathrm{~cm}^{-1}$, se forman, además, débiles bandas de absorción de los enlaces $\mathrm{Pb}-\mathrm{O}$. Mientras que cuando la recuperación de galena es óptima $75 \% \mathrm{w} / \mathrm{w}$, el azufre de la superficie se combina con el metal formando sulfatos coordinados de manera monodentada con tres bandas de absorción, así como enlaces $\mathrm{Pb}$ - $\mathrm{OH}$ del hidróxido de plomo $\mathrm{Pb}(\mathrm{OH})_{2}$ y enlaces $\mathrm{Pb}-\mathrm{O}$, del $\mathrm{PbO}_{2}$.
\end{abstract}

\section{Palabras Clave:}

Galena, flotación sin colector, depresor, oxidación, pH

Autor para la correspondencia: mreyes@uaeh.edu.mx

Correos electrónicos: mreyes@uaeh.edu.mx (M. Reyes-Pérez), alejandro.reyes@uasp.mx (I. A. Reyes-Domínguez), uri_fg@hotmail.com (M. U. Flores-Guerrero frbh68@hotmail.com (F. R. Barrientos-Hernández), miguelabra@hotmail.com (M. Pérez-Labra), jutj731101@hotmail.com (J. C. Juárez-Tapia)

ORCID: https://orcid.org/0000-0003-3843-2397 (M. Reyes-Pérez), https://orcid.org/0000-0002-6154-8294 (I. A. Reyes-Domínguez), https://orcid.org/0000-0001-9705-2306 (M. U. Flores-Guerrero), https://orcid.org/0000-0001-5459-7162 (F, R., Barrientos Hernández) https://orcid.org/0000-0001-9882-6932 (M. Pérez-Labra), https://orcid.org/0000-00017058-1670 (J. C. Juárez-tapia) 


\section{Introducción}

La galena es un sulfuro mineral de plomo $(\mathrm{PbS})$, muy abundante en todo el mundo, se encuentra asociado generalmente a los yacimientos minerales de otros sulfuros de metales base como la esfalerita $(\mathrm{ZnS})$, calcopirita $\left(\mathrm{CuFeS}_{2}\right)$, Bornita $\left(\mathrm{Cu}_{5} \mathrm{FeS}_{4}\right)$ entre otros [1, 2]. La flotación es considerada como un proceso efectivo de separación para recuperar selectivamente el sulfuro de plomo de los sulfuros polimetálicos que lo acompañan y de la ganga mineral. El éxito de la flotación, radica en la hidrofobicidad la cual es una propiedad superficial de todos los sulfuros [3]

Debido a las amplias diferencias de hidrofobicidad entre los sulfuros minerales, su separación por el método de flotación llega a ser relativamente sencilla. Para incrementar la hidrofobicidad y la selectividad hacia los minerales valiosos se emplean reactivos colectores y existe una amplia variedad de estos, se clasifican como, catiónicos y aniónicos, los más comúnmente usados tanto en la industria como en el laboratorio son los colectores aniónicos, conocidos como xantatos, clasificados en base a la longitud de su cadena hidrocarbonada [4].

En la literatura se ha discutido el tema de la flotación natural y la flotación sin colector de los sulfuros minerales [5]. Se ha demostrado que la flotación natural se debe a la formación de azufre elemental en la superficie del mineral (ejemplos de minerales con flotabilidad natural son la molibdenita, rejalgar, oropimente) [6], algunos autores, han identificado que la especie hidrofóbica en la flotación sin colector, es una superficie deficiente en metal y rica en poli sulfuros [7]. Además, la flotación sin colector también se refiere como un proceso de flotación por espuma debido a que utiliza solamente reactivo espumante, cuyo objetivo es disminuir la tensión superficial de la pulpa [2].

Los fenómenos superficiales anteriormente mencionados, mejoran sustancialmente la hidrofobicidad de los sulfuros, y hacen posible la flotación sin colector, este proceso, presenta ventajas como; la reducción o nulo consumo de reactivos químicos, el aumento de la selectividad durante la flotación, y reduce el número de equipos necesarios para lograr la óptima separación. Sin embargo, también puede tener algunos efectos negativos, como es, la adición de algunos iones metálicos al sistema de flotación para conducir a la separación de algunos minerales con poca capacidad de flotación [2].

Debido a la coexistencia de la galena con otros sulfuros minerales, la flotación es el método más empleado para concentrar esta especie mineral [8]. Para lograr eficientemente la separación de los sulfuros polimetálicos portadores de plomo, se ha reportado el uso efectivo de inhibidores o depresores de superficie, estos pueden ser orgánicos o inorgánicos tales como el dicromato de potasio, cianuro, sulfito de sodio. Sin embargo, estos dañan peligrosamente el medio ambiente, son tóxicos, venenosos y su uso cada vez es más restringido [9-11].

En otros trabajos se ha reportado el empleo de reactivos químicos orgánicos para deprimir la flotación de galena, estos son carboximetilcelulosa [12], acido húmico [13], almidón [14], tanino [15], lignina [16], estos autores, reportan un efecto depresor selectivo de la galena de otros sulfuros presentes en la pulpa mineral. En el procesamiento de los sulfuros minerales polimetálicos, el mineral de galena se flota generalmente en un segundo circuito de flotación por tal razón su superficie debe experimentar inicialmente una respuesta hidrofílica [12].

Se ha establecido previamente en la literatura que la depresión de galena se presenta a valores de $\mathrm{pH}$ mayores a [11], debido a la formación de hidróxido de plomo $\mathrm{Pb}(\mathrm{OH})_{3}{ }^{-}$, el cual se llega a retener en la superficie, además se ha mostrado que la galena muestra buena flotabilidad a pH. 8 La depresión de la galena a $\mathrm{pH}$ alcalino se atribuye a la presencia oxi hidróxidos metálicos de hierro los cuales generan una superficie hidrofílica, reduciendo así la flotabilidad, estos hidróxidos de hierro provienen de la oxidación del hierro del acero forjado de los medios de molienda y del hierro contenido en el mineral de pirita [3].

Más recientemente se ha propuesto el uso de serpentina, para deprimir los sulfuros minerales de plomo, en forma de un recubrimiento de limo hidrofílico, demostrando ser exitoso en la separación del mineral de galena de la pirita, esta depresión es controlada principalmente por la magnitud y el signo de la carga superficial de las partículas. La serpentina es un mineral de ganga comúnmente encontrado en los sulfuros minerales complejos [17].

En este trabajo de investigación se estudia el efecto del pH en la depresión del mineral de galena durante la flotación sin colector, evaluando, tanto ambientes ácidos como alcalinos, así como un amplio rango de potenciales de pulpa $E h(\mathrm{mV})$ y su efecto en el éxito o detrimento de la flotación de galena, la especiación superficial de las partículas de galena flotadas, se estudió mediante espectroscopia de infrarrojo por transformada de Fourier con la finalidad de determinar del mecanismo depresor de la galena por efecto del $\mathrm{pH}$.

\section{Desarrollo Experimental}

Para llevar a cabo el estudio del efecto depresor del $\mathrm{pH}$ en la flotación sin colector de galena, se utilizó un sulfuro mineral de plomo (PbS), el cual se muestreó y seleccionó manualmente debido a su apariencia macroscópica de estar conformado por galena, proveniente de Zimapan, Hidalgo, México coordenadas geográficas $19^{\circ} 47^{\prime} 00^{\prime}$ y y $20^{\circ} 50^{\prime} 00^{\prime}$ ' de latitud norte, y de longitud oeste $98^{\circ} 27^{\prime} 00^{\prime \prime}$ y $99^{\circ} 30^{\prime} 00$ [18]. Primero el mineral de una sola pieza se fragmentó hasta un tamaño de aproximadamente 2 milímetros, en una trituradora de quijadas de laboratorio, posteriormente se molió en un molino de $6 \mathrm{~L}$ de capacidad máxima, empleando 6 kilogramos de medios de molienda de acero forjado $66.3 \%$ de $12.5 \mathrm{~cm}$ y $33.7 \%$ de 2.54 $\mathrm{cm}$ de diámetro, durante un tiempo de molienda de 20 minutos usando $1 \mathrm{~L}$ de agua y 400 grs de mineral.

Las partículas obtenidas en la molienda se caracterizaron por: difracción de rayos $\mathrm{X}$, usando un difractometro de rayos $\mathrm{X}$ marca Bruker modelo D8, con radiación de $\mathrm{Cu} k \alpha \lambda=1.5406 \AA$. Así como por microscopia electrónica de barrido en conjunto con microanálisis por dispersión de energías en un equipo JEOL modelo JSM 5900 y por espectroscopía de infrarrojo por transformada de Fourier, en un espectrómetro Perkin Elmer modelo Spectrum GX.

Para realizar las pruebas de flotación se empleó una celda Denver de laboratorio de $1 \mathrm{~L}$, fabricada en acero inoxidable, 
mientras que el impulsor y difusor construidos de polipropileno, la velocidad de agitación del impulsor fue de 1200 revoluciones por minuto RPM, medidas con un tacómetro. El aire inyectado a la celda para formar las burbujas durante el proceso fue succionado por efecto del movimiento del impulsor hacia la celda. En todos los experimentos se utilizó agua desionizada y reactivos químicos grado analítico tal como; solución de hidróxido de sodio $1 \mathrm{M}$ para alcalinizar la pulpa y ácido sulfúrico diluido $1 \mathrm{M}$ para disminuir el $\mathrm{pH}$.

Durante el acondicionamiento de la pulpa y al final de cada una de las pruebas de flotación se monitoreó, tanto el pH como el potencial óxido reducción (con sus siglas en ingles oxide reduction potential ORP) (mV), estas variables se midieron con un potenciómetro, marca Thermo Scientific Orion 3 Star equipado con un electrodo de $\mathrm{pH}$, Ross ultra triode provisto con un sensor de temperatura.

\section{Procedimiento Experimental}

Para cada una de las pruebas de flotación se utilizó un volumen de $1 \mathrm{~L}$ de agua desionizada. el acondicionamiento de la pulpa se realizó dentro de la celda de flotación. Al inicio, con solo el agua, se midió el $\mathrm{pH}$, el potencial óxido reducción, posteriormente se adicionó el espumante $60 \mathrm{mg} / \mathrm{L}$ de metil isobutil carbinol MIBC, se dio un tiempo de dispersión, y posteriormente se agregó el sulfuro mineral de plomo, galena ( $\mathrm{PbS}) 4$ gramos, tres minutos después se ajustó el $\mathrm{pH}$ al valor predeterminado usando $\mathrm{NaOH} 1 \mathrm{M}$ para los valores alcalinos y $\mathrm{H}_{2} \mathrm{SO}_{4} 1 \mathrm{M}$ para los ambientes ligeramente ácidos, este procedimiento se repitió para cada una de las pruebas de flotación.

Después del tiempo de acondicionamiento, se inició la prueba de flotación, colectando los derrames de concentrado en tiempos de $0.5,1,2,4,6,8$ y 10 minutos en recipientes previamente pesados, la espuma se ayudó a derramar con un accesorio de material inerte. Una vez concluida la colección de la espuma conteniendo las partículas de galena flotadas, se cerró la válvula de aire, este procedimiento se repitió para cada tiempo y prueba de flotación realizada.

Los derrames de concentrado obtenidos en cada una de las pruebas y tiempo de flotación, se secaron, pesaron y por diferencia de peso del recipiente con y sin mineral se obtuvo la masa de galena flotada, calculando el porcentaje de flotación obtenido en cada uno de los tiempos, analizados con la ecuación 1. Donde Wt es la cantidad de mineral flotado en cada tiempo flotación y Wo cantidad de mineral inicial.

$$
\% \text { Flotación }=\frac{W_{t}}{W_{o}} * 100
$$

Los sólidos de galena más significativos se caracterizaron mediante espectroscopia de infrarrojo por transformada de Fourier con la finalidad de identificar las especies covalentes presentes en la superficie del mineral de galena, y así determinar la especiación superficial y explicar el mecanismo de depresión o activación del mineral durante la flotación.

\section{Resultados y discusión}

\section{Caracterización}

La figura 1 presenta el patrón de difracción de rayos $\mathrm{X}$ de la muestra mineral de galena, se observaron dos fases mineralógicas en el mineral, sulfuro de plomo $\mathrm{PbS}$ y sulfuro de zinc ZnS.

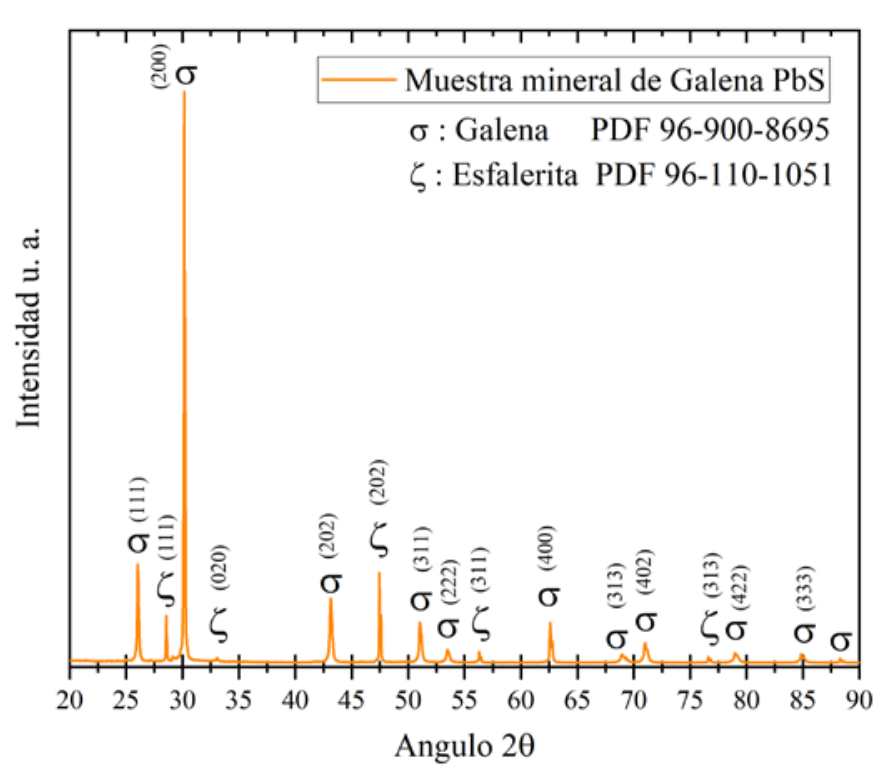

Figura 1. Espectro de difracción de rayos $X$ del mineral utilizado para las pruebas de flotación.

Siendo la galena la fase mayoritaria detectada. Esto indica un mineral de pureza considerable constituida mayormente por galena. El espectro obtenido por difracción por DRX se indexó usando el software Match 1.1, los patrones de difracción PDF que coinciden con la muestra mineral son 96-900-8695 para la galena y 96-110-0044 para la esfalerita.

La figura 2 muestra las imágenes de MEB a detalle de una partícula de galena y el microanálisis por dispersión de energías de la superficie libre de micro partículas espectro 1 y de estas, espectro 2. Los resultados concuerdan con lo encontrado en DRX, donde además de los componentes de la galena se detectó al zinc.

De los microanálisis por EDS de la superficie de la galena se tiene, que es rica en plomo con $81.67 \%$ y pobre en azufre y zinc con porcentajes de $10.61 \%$ y $7.72 \%$ respectivamente. Mientras que una de las micro partículas presente en la superficie de la galena, espectro 2, muestra menor contenido de plomo $79.89 \%$, e incrementa el azufre y zinc en $11.4 \%$ y $8.97 \%$ respectivamente. Cabe mencionar que los EDS son microanálisis semi cuantitativos, y difieren de la cantidad estequiométrica de un mineral puro de galena y esfalerita.

La figura 3 presenta el espectro de infrarrojo representativo de las partículas de galena $<74 \mu \mathrm{m}$ usadas para todas las pruebas de flotación. Se observan múltiples bandas de absorción. La 
identificación de estas, se apoyó en la obtención de los espectros de FTIR de reactivos químicamente puros de $\mathrm{PbO}_{2}$ y $\mathrm{PbO}$. De aquí, que las bandas en $420 \mathrm{~cm}^{-1}, 462 \mathrm{~cm}^{-1}, 595 \mathrm{~cm}^{-1}$ y $619 \mathrm{~cm}^{-}$ ${ }^{1}$ corresponden a los modos de vibración de los enlaces $\mathrm{Pb}-\mathrm{O}$ del óxido de plomo II $\left(\mathrm{PbO}_{2}\right)$. Y las señales en $693 \mathrm{~cm}^{-1}$ y 796 $\mathrm{cm}^{-1}$ corresponden al enlace $\mathrm{Pb}-\mathrm{O}$ del óxido de plomo $\mathrm{PbO}$.
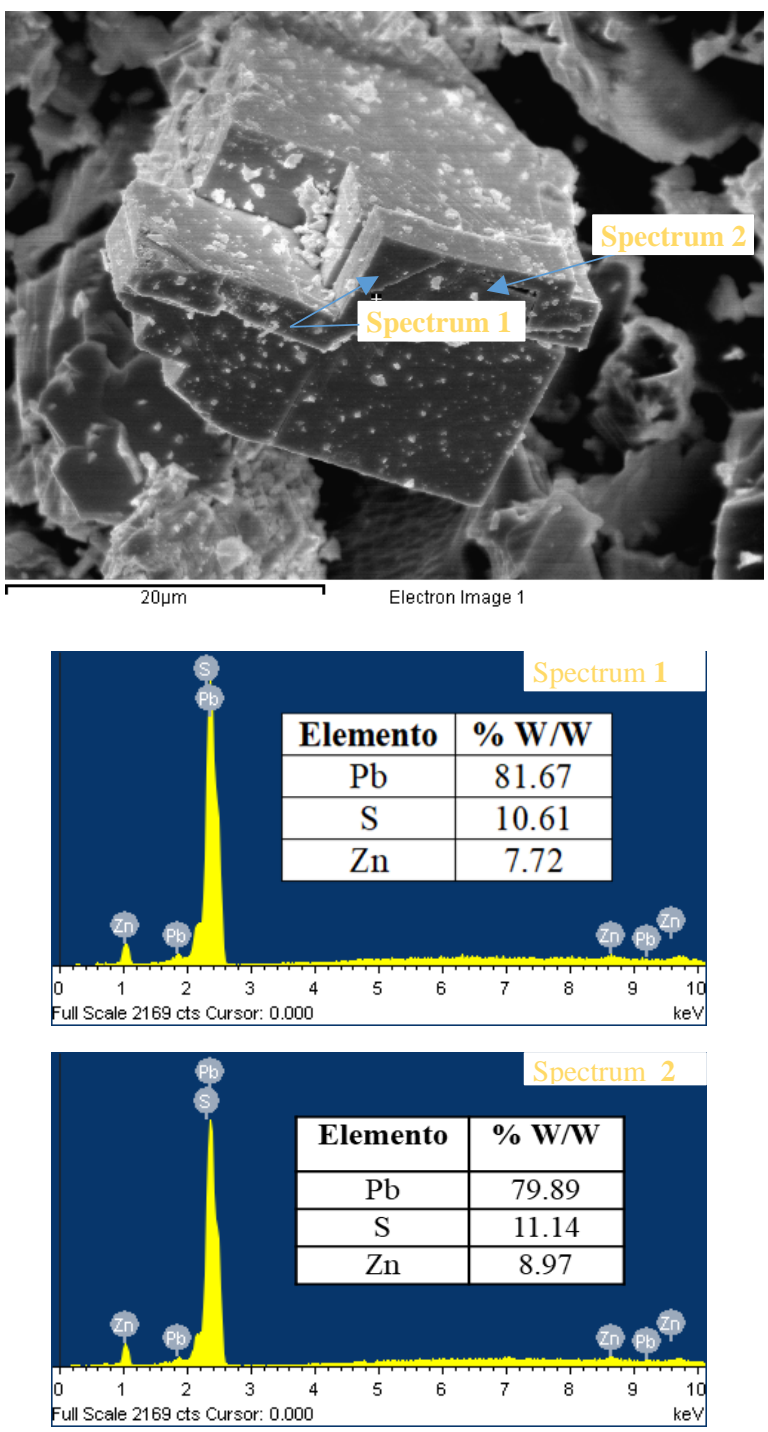

Figura 2. Imagen de MEB y Micro análisis semi cuantitativo puntual por EDS de la galena

Por otro lado, las bandas en $1054 \mathrm{~cm}^{-1}$ y $1112 \mathrm{~cm}^{-1}$ y el débil hombro de absorción en $967 \mathrm{~cm}^{-1}$, indican la división de la banda principal del ion sulfato en tres bandas de absorción indicando que el ion sulfato se combina con el plomo formando un compuesto de sulfato plomo, señales de absorción que han sido descritas en trabajos previos [19-21].

\section{Flotación sin colector de galena (PbS)}

En la literatura se menciona que la galena es casi totalmente flotable sin colector en condiciones anaeróbicas y en la flotación del mineral por separado [22]. Industrialmente en el proceso de concentración Tsumeb, en Sudáfrica, se aplica la flotación sin colector para la separación selectiva de galena y esfalerita de un mineral conteniendo calcopirita [23]. De documentos previos se ha establecido que el azufre elemental es una especie muy hidrofóbica y su presencia puede causar la flotabilidad de los sulfuros sin colector [24].

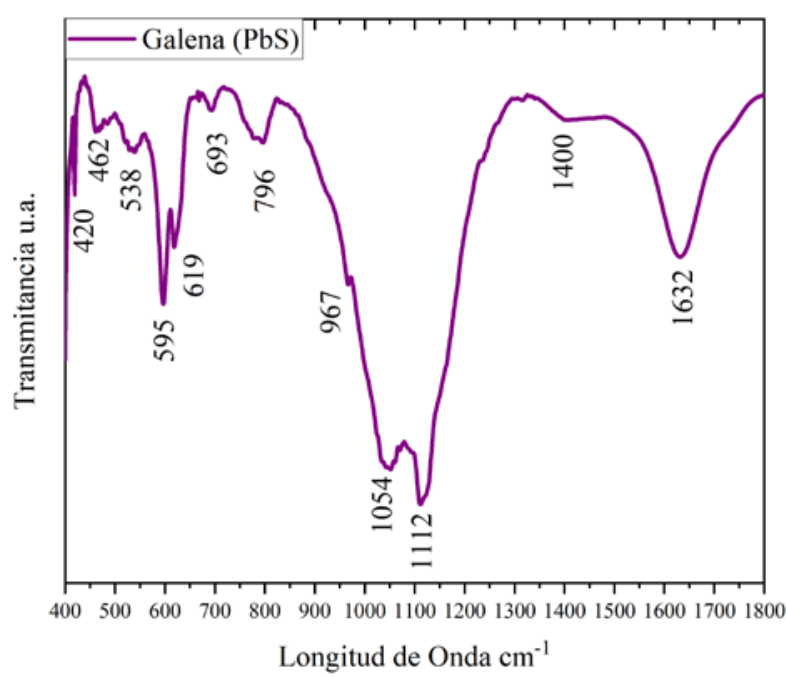

Figura 3 Espectro de Infrarrojo de galena producto de la molienda con medios de acero.

La figura 4 muestra los resultados obtenidos en la flotación sin colector de galena Vs. El tiempo (minutos) para valores de $\mathrm{pH}$ ligeramente ácidos, neutros y alcalinos. La flotación de galena es óptima, cuando el pH de la pulpa es ácido 5.67 y 6 logrando alrededor del 75 \% W/W de separación, además, se observa en la figura 4 que cuando se han cumplido cuatro minutos de flotación se ha alcanzado alrededor del $65 \%$ de flotación acumulativa. No obstante, la presencia de esfalerita en la muestra mineral en bajas proporciones, el objetivo de este trabajo se enfocó solamente en el efecto depresor del pH sobre el mineral de galena.

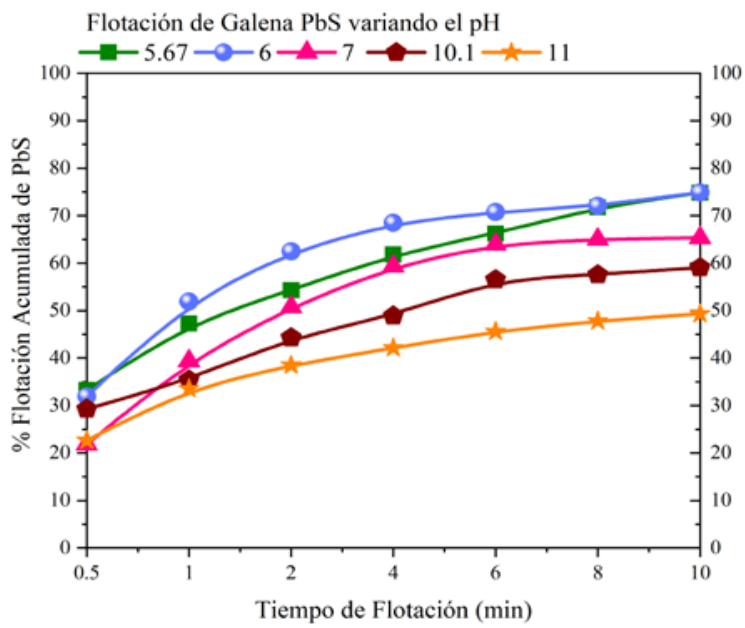

Figura 4. \% Flotación acumula de PbS en función del tiempo (minutos) efecto del $\mathrm{pH}$.

Por otro lado, la flotación sin colector de galena se deprime significativamente con el aumento del $\mathrm{pH}$ y en este estudio la inhibición de la flotación es máxima cuando el pH es 11, un 25 $\%$ menos que la obtenida cuando el pH de flotación es ácido. 
De estudios previos se han encontrado resultados similares indicando la depresión de galena a valores de $\mathrm{pH}$ arriba de 11 . Los autores indican que la depresión del mineral de galena es debido a la formación de hidróxidos de Plomo particularmente $\mathrm{Pb}(\mathrm{OH})_{3}{ }^{-}$los cuales eventualmente se retienen en la superficie de la galena [25].

De la medición del pH se encontró que al final de la prueba de flotación sin colector de galena el $\mathrm{pH}$ de la pulpa tiende a disminuir, esto se atribuye directamente a la oxidación de los elementos que componen a la galena, las ecuaciones 2 y 3 muestran este comportamiento, y debido a que tienden a generar ion hidrogeno $\left(\mathrm{H}^{+}\right)$, provocara la disminución del carácter alcalino de la pulpa, tal como se encontró en las mediciones realizadas.

$$
\begin{gathered}
\mathrm{Pb}^{2+}+\mathrm{H}_{2} \mathrm{O} \rightarrow \mathrm{Pb}(\mathrm{OH})^{+}+\mathrm{H}^{+} \\
\mathrm{S}^{-1}+4 \mathrm{H}_{2} \mathrm{O} \rightarrow \mathrm{SO}_{4}^{-2}+8 \mathrm{H}^{+}+7 \mathrm{e}^{-}
\end{gathered}
$$

Durante la flotación de galena sin colector se midió el potencial óxido reducción $(\mathrm{mV})$, y los valores se grafican en referencia al electrodo estándar de hidrógeno Eh (mV). La figura 5 muestra los valores de potencial obtenidos durante el acondicionamiento, al inicio y al final de la prueba de flotación.

De esta figura se observa que, después de la adición del espumante [e] $=60 \mathrm{mg} / \mathrm{L}$ y del mineral se tiene un potencial Eh de $+275 \mathrm{mV}$ con un $\mathrm{pH}$ de flotación de 5.67 y 6 . En estos valores de $\mathrm{pH}$ se obtuvieron los mayores porcentajes de flotación acumulativa de galena $(\mathrm{PbS}), \mathrm{y}$ se ve significativamente favorecida cuando se tienen potenciales positivos de $+325 \mathrm{mV}$.

Por otro lado, la disminución del carácter oxidante de la pulpa de flotación a valores de potencial Eh menores a $+100 \mathrm{mV}$ se afecta sustancialmente la eficiencia de flotación resultados similares se presentan en la literatura 3, no obstante, de estas condiciones no se deprime totalmente la galena logrando un porcentaje de flotación acumulada de $50 \%$ a pH 11 . Es de esperar que los cambios en el potencial Eh (mV) alteren la naturaleza superficial de galena y estas variaciones de potencial influyen directamente en el éxito o fracaso de la flotación de galena.

De la figura 4 se observa que el potencial pierde la dependencia del $\mathrm{pH}$ esto es debido a las reacciones oxido reducción que suceden entre las especies minerales y el medio. Los valores del potencial de pulpa obtenidos en este trabajo para una sola especie mineral, se espera varíen en una pulpa industrial debido a la multitud de especies que sufren el proceso oxido reducción.

El pH es una variable crítica e influye tanto en la eficiencia de flotación, como en el potencial de pulpa $E h(\mathrm{mV})$ y en la producción de especies oxidadas en las superficies de las partículas del sulfuro de plomo. El estado superficial de las partículas de mineral de galena flotadas en diferentes valores de pH se analizó vía espectroscopia de infrarrojo por transformada de Fourier FTIR.

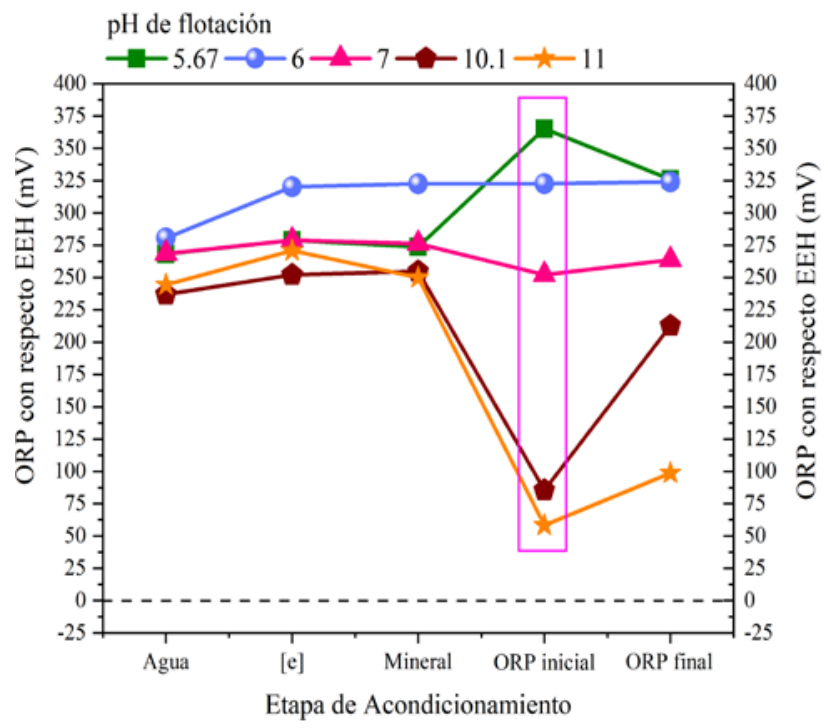

Figura 5. Potencial óxido reducción referido al Electrodo Estándar de Hidrogeno ( $\mathrm{mV}$ ) Vs. La etapa de acondicionamiento.

La figura 6 muestra los espectros de infrarrojo de la galena flotada a pH inicial 11.0 obtenidas en tiempos de flotación de 0.5 y 10 minutos, cabe recordar que en este valor de $\mathrm{pH}$ se obtuvo la recuperación de galena más baja. Ambos espectros mantienen mucha similitud. Predomina del resto de las bandas de absorción, la señal en $1097 \mathrm{~cm}^{-1}$ lo cual indica la presencia de ion sulfato libre en la superficie del mineral, se observa además un débil hombro de absorción que puede indicar el inicio de la escisión de la banda principal del ion sulfato en 3 y 4 bandas dividiendo la banda principal $\mathrm{C}_{3 \mathrm{v}}$ dichas bandas se coordinan con el metal $(\mathrm{Pb})$.

Se observa además una fuerte banda de absorción en alrededor de $1400 \mathrm{~cm}-1$ atribuida a la presencia de enlaces del tipo azufre - oxígeno $\mathrm{S}=\mathrm{O}$ resultado de la quimio absorción del oxígeno molecular con el azufre superficial en el mineral de galena. En lo que respecta a los iones hidroxilo adsorbidos en la estructura superficial de galena presenta una banda de absorción típica en $1638 \mathrm{~cm}^{-1}$ indiscutiblemente la superficie de galena sufre una oxidación parcial considerable. Las bandas detectadas en 418 $\mathrm{cm}^{-1}, 460 \mathrm{~cm}^{-1}$ y $617 \mathrm{~cm}^{-1}$ se asignan a los modos de vibración de los enlaces $\mathrm{Pb}$ - $\mathrm{O}$ del dióxido de Plomo $\mathrm{PbO}_{2}$ mientras las bandas en $681 \mathrm{~cm}^{-1}, 779 \mathrm{~cm}^{-1}$ y $1736 \mathrm{~cm}^{-1}$ corresponden a las vibraciones de los enlaces covalentes $\mathrm{Pb}$ - $\mathrm{O}$ del $\mathrm{PbO}$.

La figura 7 muestra los espectros de infrarrojo de la galena flotada a pH 5.67. La banda de absorción en $1632 \mathrm{~cm}^{-1}$, se asigna al modo de flexión de las moléculas de agua absorbidas a la superficie de la galena, formando el grupo $\mathrm{Pb}-\mathrm{OH}$, cabe citar que la intensidad de esta banda es mucho más grande a las obtenidas cuando se lleva a cabo la flotación a pH alcalino. La banda en $1380 \mathrm{~cm}^{-1}$ se asocia con la presencia de enlaces $\mathrm{S}=\mathrm{O}$ y se encuentra en menor intensidad respecto a los espectros de la flotación realizada a pH 11 figura 6. 


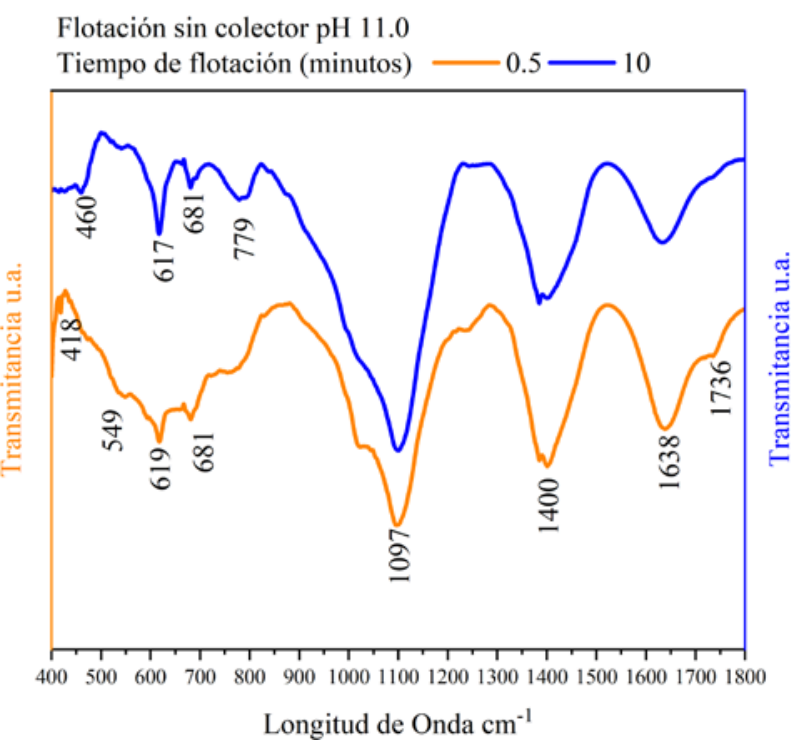

Figura 6. Espectros de Infrarrojo de galena flotada a $\mathrm{pH} 11$ en 0.5 y 10 minutos.

La zona espectral más interesante es la que se encuentra entre $1000 \mathrm{~cm}^{-1}$ y $1200 \mathrm{~cm}^{-1}$ correspondientes a las especies azufre en especial al ion sulfato, se observa en la figura 7 , especialmente para el espectro de 0.5 minutos, la banda principal del ion sulfato sufre la división asimétrica con tres bandas de absorción en $1036 \mathrm{~cm}^{-1}, 1105 \mathrm{~cm}^{-1}$ y $1124 \mathrm{~cm}^{-1}$ indicando la coordinación del sulfato con el plomo en forma de compuestos monodentados situación similar se ha descrito en la literatura [19, 20].

La banda de absorción en $790 \mathrm{~cm}^{-1}$ se asocia con la presencia de enlaces $\mathrm{Pb}$ - $\mathrm{OH}$ del oxi hidróxido de plomo por lo que la mejor respuesta a la flotación o el mecanismo por el cual se obtiene mejor eficiencia de flotación es la debido a la presencia de las especies tipo sulfato y oxi hidróxido de plomo descritas anteriormente. Las bandas correspondientes a los enlaces $\mathrm{Pb}$ $\mathrm{O}$ del $\mathrm{PbO}$ y $\mathrm{PbO}_{2}$ localizadas en $594 \mathrm{~cm}^{-1}$ y $618 \mathrm{~cm}^{-1}$ respectivamente. Por lo que la disminución tanto de intensidad como de numero de bandas de absorción de enlaces $\mathrm{Pb}-\mathrm{O}$ del $\mathrm{PbO}$ y $\mathrm{PbO}_{2}$ mejoraran significativamente la flotación de galena.

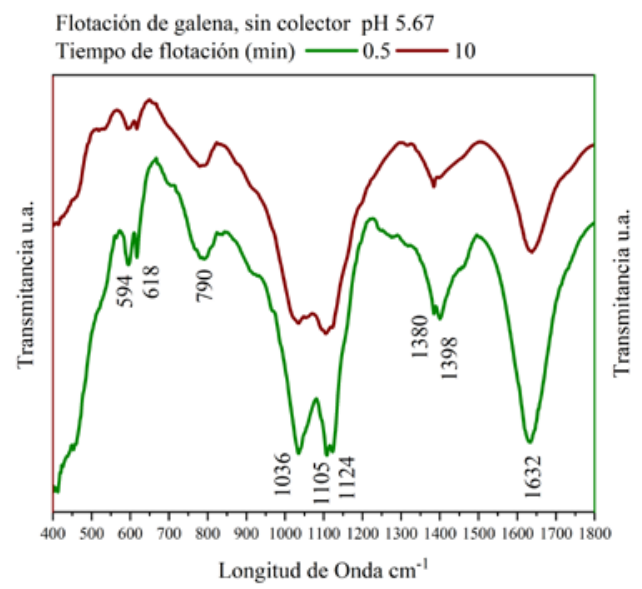

Figura 7. Espectros de Infrarrojo de galena flotada a pH 5.67 0.5 y 10 minutos

\section{Conclusiones}

En este trabajo de investigación, se estudió el papel depresor del $\mathrm{pH}$ durante la flotación sin colector del sulfuro mineral de plomo $\mathrm{PbS}$, la caracterización del mineral de galena objeto de estudio indicó un mineral tipo de galena con contenidos minoritarios de esfalerita. El pH de la pulpa de flotación influye significativamente, de esta manera, la galena se deprime en ambientes neutros y alcalinos, pero en ambientes ligeramente ácidos la superficie de galena se activa logrando porcentajes acumulativos de flotación del $75 \%$ w/w en un tiempo de 10 minutos, las especies responsables del éxito de la flotación, detectadas en la superficie del mineral por FTIR, fue, el ion sulfato coordinado con el plomo de manera monodentada con tres bandas de absorción, enlaces $\mathrm{Pb}-\mathrm{OH}$, del hidróxido de plomo $\mathrm{Pb}(\mathrm{OH})_{2}$ y $\mathrm{Pb}-\mathrm{O}$ del $\mathrm{PbO}_{2}$. Mientras que la depresión de la galena se da por la presencia del ion sulfato libre en la superficie, así como el incremento de la intensidad de los sulfo óxidos $\mathrm{S}=\mathrm{O}$ y la presencia de múltiples bandas de enlace de los enlaces $\mathrm{Pb}-\mathrm{O}$ del $\mathrm{PbO}$ y $\mathrm{PbO}_{2}$.

\section{Conflicto de intereses}

Los autores declaran que no existen conflictos de intereses.

\section{Referencias}

[1] Worner H K, Mitchell R W. (Editors), Minerals of Broken Hill, Australian Mining \& Smelting Limited, Griffin Press, Melbourne, Australia, editors.

[2] Feng Q, Chen J. Electrochemistry of Sulfide Minerals Flotation. Central South University Press, editors.

[3] Sajjad A, Seyed K M, Mahdi G. Chemical and colloidal aspects of collectorless flotation behavior of sulfide and non-sulfide minerals. Advances in Colloid and Interface Science 2015; 225: 203-217.

[4] Rao S R, Finch J A. Base metal oxide flotation using long chain xanthates. Int. J. Miner. Process 2003; 69: 251- 258.

[5] Fuerstenau M C., Misra M, Palmer B R. Xanthate adsorption on selected sulphides in the presence of oxygen. Inter. J. Miner. Process 1980; 29I (12): 111-119.

[6] Finkelstein N P, Allsion S A, Lovell V M, Stewart B V. Advances in Interf Pheno. of Particulate/Solution/Gas Systems (Eds. P. Somasundaran and R. B. Grieves). AIChE: New York. 1975; 71(150): 165- 175.

[7] Buckley A N, Hamilton I C, Woods R. Investigation of the surface oxidation of sulphide minerals by linear potential sweep and X-ray photoelectron. In: K. S. E. Forssberg (ed.), Flotation of Sulphide Minerals, Elsevier. Amsterdam 1985; 6: 41- 60.

[8] Xingjie W, Wenqing Q, Fen J, Ruizeng L, Daowei W. Inhibition of galena flotation by humic acid: Identification of the adsorption site for humic acid on moderately oxidized galena surface. Minerals Engineering 2019; 137: 102-107.

[9] Bolin N, Laskowski J. Polysaccharides in flotation of sulphides. Part II. Copper/ lead separation with dextrin and sodium hydroxide. Int. J. Miner. Process. 1991; 33: 235-241.

[10] Bulatovic S, Wyslouzil D M. Selection and evaluation of different depressants systems for flotation of complex sulphide ores, Miner. Eng. 1995; 8 (1-2): 63-76.

[11] Sarquís P E, Aguado J M, Mahamud M M, Dzioba R. Tannins: the organic depressants alternative in selective flotation of sulfides, J. Clean. Prod. 2014; 84 (1): 723-726.

[12] Valdivieso A, Ledesma L A, Cabrera A, Navarro O A. Carboxymethylcellulose (CMC) as PbS depressant in the processing of $\mathrm{Pb}$ $\mathrm{Cu}$ bulk concentrates. Adsorption and floatability studies. Miner. Eng. 2017; 112: 77-83. 
[13] Wang D, Jiao F, Qin W, Wang X. Effect of surface oxidation on the flotation separation of chalcopyrite and galena using sodium humate as depressant. Sep. Sci. Technol. 2017; 53: 961-972.

[14] Kar B, Sahoo H, Rath S S, Das B. Investigations on different starches as depressants for iron ore flotation. Miner. Eng. 2013; 49: 1-6.

[15] Sarquís P E, Menéndez A, Mahamud J, Dzioba R. Tannins: the organic depressants alternative in selective flotation of sulfides. J. Clean. Prod. 2014; 84: 723-726.

[16] Liu Q, Laskowski J S. The role of metal hydroxides at mineral surfaces in dextrin adsorption, II. Chalcopyrite galena separations in the presence of dextrin. Int. J. Miner. Process. 1989; 27: 147-155.

[17] Bo F, Wenpu Z, Yutao G, Tao W, Guodong L, Huihui W, Guichun H. The flotation separation of galena and pyrite using serpentine as depressant. Powder Technology 2019; 342: 486-490.

[18] J. Solana López. Servicio Geológico Mexicano, Fideicomiso de fomento minero. Inventario físico de los recursos minerales del municipio Zimapán, Estado de Hidalgo. 2008

[19] Nakamoto K. Infrared and Raman Spectra of Inorganic and Coordination Compounds., New York, Wiley, editors.

[20] Hug S J. In Situ Fourier Transform Infrared Measurements of Sulfate Adsorption on Hematite in Aqueous Solutions. Journal of Colloid and Interface Science 1997; 188(2): 415-422.

[21] M. Reyes P. (2013) Modificación superficial de mineral de pirita y precipitados de Hierro: comportamiento en medios acuosos y de molienda. UMSNH, editor.

[22] Yuehua H, Wei S, Dianzuo W. Electrochemistry of flotation of sulphide minerals. Tsinghua University Press. Springer, editors.

[23] Sun S, Li B, Wang D. Collectorless flotation of sulphide minerals. J. Cent. South Inst. Min. Metall. 1990; 21(5): 473 - 478.

[24] Fornasiero D, Ralston J. Effect of surface oxide/hydroxide products on the collectorless flotation of copper activated sphalerite. International Journal of Mineral Processing 2006; 78(4): 231-237.

[25] Ekmekçi, Z. and H. Demirel, Effects of galvanic interaction on collectorless flotation behavior of chalcopyrite and pyrite. International journal of mineral processing, 1997. 52(1): p. 31-48. 